\title{
Skilled Reaching Deficits in Unilateral Dopamine-depleted Rats: Impairments in Movement and Posture and Compensatory Adjustments
}

\author{
Elena I. Miklyaeva, Eddie Castañeda, and lan Q. Whishaw \\ Department of Psychology, University of Lethbridge, Lethbridge, Alberta, Canada T1K 3M4
}

Rats receiving unilateral dopamine (DA) depletions have bilateral deficits in using the forelimbs for skilled reaching. These impairments were investigated using end-point, video, and kinematic measures. Control rats and groups of rats with $>98 \%$ unilateral depletions (confirmed by tissue, apomorphine, and amphetamine assay), produced by 6-hydroxydopamine (6-OHDA) injected into the nigrostriatal bundle, were tested for $100 \mathrm{~d}$ on two reaching tasks. One depleted group used the contralateral-to-lesion (bad), and the other the ipsilateral-to-lesion (good), limb. Rats using their bad limb made few successful reaches and had dysfunctions in aiming, pronating, and supinating, and in using the digits to grasp and release food. Rats using the good limb were initially impaired, but improved, and could make these movements. Neither depleted group was able to use their bad limbs to assist actively the postural changes required for reaching. Thus, they did not use the diagonal supporting pattern (the nonreaching forelimb and diagonal hind limb) from which control rats initiated reaching. The group using their bad limb initiated reaching from a base of support distributed on the ipsilateral-to-lesion limbs, while the group using their good limb initiated reaching from a base of support centered on the ipsilateral rear limb. The impairments in making voluntary movements and postural adjustments with the bad limbs and the behavioral changes introduced by the compensatory dependency on good limbs account for the bilateral deficits in skilled movements. The results show that DA is required for skllled movements and postural adjustments and demonstrate that behavioral compensation can contribute to recovery.

IKey words: dopamine and behavioral compensation, dopamine and posture, dopamine and skilled reaching, dopamine and voluntary movement, rat limb and posture, rat skilled reachingl

Animals with extensive bilateral depletion of striatal dopamine (DA), produced by the neurotoxin 6-hydroxydopamine (6OHDA) injected into the lateral ventricles or nigrostriatal bundle, are seriously compromised in their ability to walk (Schallert et al., 1978), orient to ambient sensory events or stimuli that

\footnotetext{
Received Feb. 25, 1994; revised Apr. 25, 1994; accepted May 26, 1994.

This research was supported by a grant from the Medical Research Cuuncil of Canada. E.I.M. was supported by a grant from the Alberta Heritage Foundation for Medical Research.

Correspondence should be addressed to Dr. I. Q. Whishaw at the above address.

Copyright (C) 1994 Society for Neuroscience $0270-6474 / 94 / 147148-11 \$ 05.00 / 0$
}

touch their body surface (Marshall et al., 1974), and perform in learning tasks (Ranje and Ungerstedt, 1977; Whishaw and Dunnett, 1985). They even fail to eat and drink and thus must be fed by gastric intubation (Ungerstedt, 1971; Zigmond and Stricker, 1973). Some behaviors are spared. Body weight is supported against gravity, displacement from a base of equilibrium is actively resisted, and normal postural adjustments are made when equilibrium is lost (Schallert et al., 1978, 1979). Since these symptoms are very similar to those displayed by human Parkinson patients (Martin, 1967; Sacks, 1973), this animal analog is used to model the human condition for the purposes of studying recovery or restitution (Schultz, 1982; Robinson et al., 1990).

Unilateral DA depletion results in less pronounced behavioral deficits in that animals can walk, eat and drink, and so on. Nevertheless, they show behavioral asymmetries, illustrated by hemispatial neglect toward, and turning biases away from, the side of their body contralateral to the lesion (Ungerstedt and Arbuthnott, 1970; Schallert et al., 1982; Carli et al., 1985; Schallert and Hall, 1988; Whishaw and Tomie, 1988). Also, they have a severe impairment in using the contralateral-to-lesion limb for skilled movements and, as a consequence, use the other limb to reach for food or bar press (Siegfried and Bures, 1980; Uguru-Okorie and Arbuthnott, 1981; Evenden and Robbins, 1984; Hamilton et al., 1985; Sabol et al., 1985; Whishaw et al., 1986), as has also been reported for human Parkinson patients (Bennett et al., 1993). In general, it has been thought that unilateral lesions do not result in impairments ipsilateral to the lesion, but impairments have been reported in skilled reaching using the ipsilateral limb (Whishaw et al., 1986, 1992a).

In nearly all of the animal studies, and especially those of skilled movements, performance has been measured in terms of end point measures, that is, success or failure. For skilled limb performance, only cursory description of behavior has been provided with no analysis of the underlying causes of poor performance. Thus, the basis of neither the deficits contralateral to the lesion nor those that occur ipsilateral to the lesion is understood. The purpose of the present study was to investigate the basis of the bilateral impairment of unilaterally DA-depleted rats in skilled reaching for food using end-point measures as well as descriptive and kinematic methods (Whishaw and Pellis 1990; Whishaw et al., 1994). Separate groups of unilateral DAdepleted rats were given lesions either ipsilateral or contralateral to their preferred limb and they were then forced to use this limb over a $100 \mathrm{~d}$ recovery period. Descriptive and kinematic analyses were done from conventional and high-speed video recordings of body, limb, and digit movements. 


\section{Material and Methods}

\section{Subjects}

Eighteen adult female, Long-Evans rats, weighing $200-230 \mathrm{gm}$ when the study began, were used. They were housed in hanging wire mesh cages in a room lighted on a 12:12 hr light/dark cycle with temperature set at $22^{\circ} \mathrm{C}$. Testing was done during the light portion of the cycle. The rats were food deprived to $90 \%$ normal body weight and received oncea-day supplemental feeding of measured amounts of food to maintain deprivation.

\section{Reaching tasks}

The rats were tested in three different tasks. In the first test, they reached to take food from their mouth (so that they could sit back on their haunches to eat as the food was held in their paws); in the second test, they reached for food that was located in a food tray; in the third test, they reached for single food pellets located on a shelf.

(1) Spontaneous grasping. A $750 \mathrm{mg}$ food pellet was placed on the floor of a box (the box used in task 3 , below) and the rat was filmed as it picked the food up with its mouth, transferred it to its paws, and then handled it as it ate (Whishaw et al., 1992b).

(2) Food tray task. The food tray task (Whishaw et al., 1993) used nine identical boxes $(10 \times 18 \times 10 \mathrm{~cm}$ high), which permitted training of a number of animals at once. The front and floor were constructed of $2 \mathrm{~mm}$ bars that were separated from each other by $9 \mathrm{~mm}$, edge to edge. The tops, backs, and sides were Plexiglas. A 4-cm-wide and 5-mmdeep tray, containing granules of food (20-40 mg chick feed), was mounted in front of each box and extended for the length of the box. To obtain food, the rat had only to reach through the bars, grasp and retract it. The floor of the boxes was made of metal grids, so that if a rat dropped the food, the food fell through the grids and was lost. Performance was scored by depressing buttons, connected to relays and then to a microcomputer, indicating hits and misses for each limb. If the rats made a reaching movement in which a paw was inserted through the bars of the cage, the movement was scored as a "reach." If the rat obtained a piece of food and then consumed it, the reach was scored as a "hit."

(3) Single pellet task. The box in which the animals reached for a single food pellet was $25 \mathrm{~cm}$ wide, $35 \mathrm{~cm}$ long, and $30 \mathrm{~cm}$ high, made of clear Plexiglas, and had an underlying inclined mirror so that the rats could be filmed from a ventral perspective (Whishaw and Pellis, 1990). Five centimeters from the side of each front wall was a 1 -cm-wide slit that extended from the floor to a height of $15 \mathrm{~cm}$. On the outside of the wall, in front of each slit, mounted $3 \mathrm{~cm}$ above the floor, was a 2-cm-wide by 4 -cm-long shelf. Food pellets ( 20 and $94 \mathrm{mg}$ Rodent Chow food pellets, Bioserve Inc., Frenchtown, NJ) were placed in one of two small indentations on the floor of the shelf. The indentations were 2 $\mathrm{cm}$ away from the inside wall of the box and were centered on the edges of the slit through which the rats reached. For each rat, food was placed in the indentation contralateral to the limb with which the rat reached. Training was administered in such a way that when a rat made a successful reach, a short pause preceded presentation of the next food pellet, during which another food pellet could be dropped into the back of the box. This encouraged the rat to leave the food aperture and then reposition itself for the next food pellet. When the rats were well trained, they were filmed from lateral, ventral, and frontal views.

\section{Videorecording}

Conventional videorecording was done with a Sony Video 8 CCD-VII portable camera (30 frames/sec) using a shutter speed of $1 / 1000$ of a second. Illumination was provided by a two-arm Nikon MII cold light source. A frecze framc option on a Sony Vidco 8 recorder was used to examine behavior on successive individual video frames.

High-speed videorecording of paw and digits movements were made with a Peak Performance Technologies Inc. 120 Video Camera (60 frames $/ \mathrm{sec}$ ), using shutter speed of $1 / 1000$ of a second. Using a freeze frame option, behavior was examined on successive video frames $(60$ frames/sec). In addition, a framegrabber was used to capture and freeze individual fields in order to examine behavior on successive fields, thus providing a resolution of 120 fields/sec.

\section{Amphetamine- and apomorphine-induced rotation}

After $60 / 70$ postsurgical days, rats were placed into one of the four automated rotometer bowls (Ungerstedt and Arbuthnott, 1970) for a
30 min adaption period before different tests in which they were given either $d$-amphetamine sulfate $(5 \mathrm{mg} / \mathrm{kg}, \mathrm{i} . \mathrm{p}$.) or apomorphine hydrochloride $(0.1 \mathrm{mg} / \mathrm{kg}$, i.p.). Turns in the ipsilateral and contralateral direction were counted via a microcomputer for $90 \mathrm{~min}$ after amphetamine and $30 \mathrm{~min}$ after apomorphine, and using previously published criteria, the turning bias was used as one of the measures of lesion size (Marshall and Ungerstedt, 1977; Hefti et al., 1980).

\section{Movement scoring}

The various movements comprising a reach have been identified previously (Whishaw and Pellis, 1990; Whishaw et al., 1991, 1993, 1994) using a conceptual framework derived from Eshkol-Wachman Movement Notation (Eshkol and Wachmann, 1958). Additionally, for the present study, a scoring system for posture was developed.

Limb movements. (1) Limb lift: the limb is lifted from the floor with the upper arm and digits being swung to the midline of the body. (2) Digits close: as the limb is lifted, the digits are semiflexed and the paw is supinated so that the palm faces the midline of the body. (3) Aim: using the upper arm, the elbow is adducted so that the forearm is aligned along the midline of the body, with the paw localed just under the mouth. This movement involves fixation of the distal portion of the limb, so that the digits remain aligned with the midline of the body. This is likely produced by a movement around the elbow that reverses the direction of movement of the paw to compensate for the adduction of the elbow. (4) Advance: the head is lifted and the limb is advanced directly forward above and beyond the food pellet. (5) Digits open: as the limb is advanced the digits are extended and opened. (6) Pronate: using a movement of the upper arm, the elbow is abducted pronating the paw over the food. Full pronation of the paw onto the food is aided by a movement of the paw around the wrist. (7) Grasp: as the pads of the palm or the digits touch the food, the food is grasped by closure of the digits. This can occur as an independent movement or the grasp can occur as the paw is withdrawn. (8) Supination I: as the limb is withdrawn, the paw is dorsiflexed and is supinated by $90^{\circ}$ by a movement around the wrist and by adduction of the elbow. These movements can occur as soon as the food is grasped or can occur as the limb is withdrawn. (9) Supination II: as the rat sits back with the food held in the paw, the paw is supinated by a further $90^{\circ}$ and ventroflexed to present the food to the mouth. (10) Food release: the digits are opened and the food is transferred to the mouth.

Each movement was rated on a three-point scale. If the movement appeared normal, the movement was given a score of 0 , if the movement appeared slightly abnormal but was recognizable, it was given a score of 1 , and if the movement was absent or compensated for entirely by movement of a different body part, the movement was given a score of 2.

Postural adjustments. A reach was divided into five different components in order to score posture. (1) Angle of orientation: this was the angle at which the rat approached the slot in order to initiate a reach. A score of 0 indicated a direct approach; 1 , an angle up to $45^{\circ}$; and 2 , a larger angle. (2) Reaching posture: if a rat lifted a single forelimb to reach, while the other limbs supported posture, it received a score of 0 ; if it placed the nonreaching forelimb on the wall or shelf, it received a score of 1 ; and if it additionally displayed abnormal placement of the other limbs or exaggerated body rotation, it received a score of 2 . (3) Limb advancement: if the rat made a single discrete reach with the appropriate limb, it received a score of 0 ; if it used its other limb in combination with the appropriate limb, or alternated limbs, it received a score of 1 ; and if it made more attempts with any combination of limbs it received a score of 2. (4) Postural corrections during reaches: if a rat initiated a reach without a major change in initiating posture, it received a score of 0 ; if it made one postural adjustment before completing a reach, or initiating a second reach, it received a score of 1 ; and if it made a number of postural corrections it received a score of 2. (5) Withdrawal and food release: if the rat withdrew the limb and placed food in its mouth or sat back to place the food in its mouth, it received a score of 0 . If it ate the food with the paw held on the shelf or floor, or if it had difficulty withdrawing the limb, it received a score of 1 , and if it dropped the food or neglected it, it received a score of 2 .

\section{Kinematics}

The Video 8 film was copied onto a VHS format and was digitized using a Peak Performance System (Englewood, $\mathrm{CO}$ ) and frame grabber at 60 fields $/ \mathrm{sec}$. The data points obtained from the computer analysis 

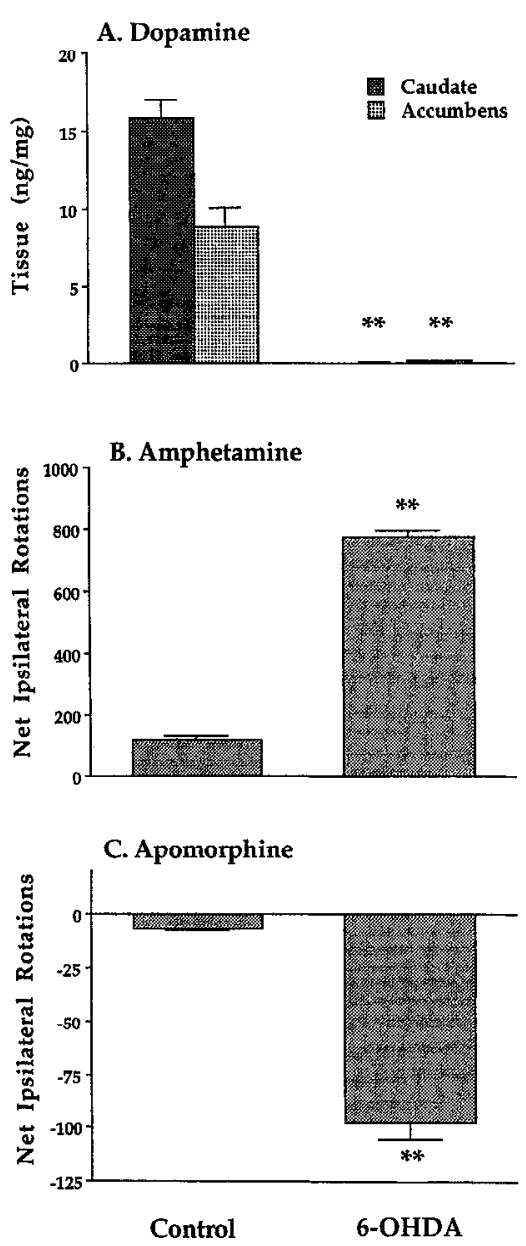

Figure 1. Lesion assessment (mean and SE) in control rats and the combined ipsilateral and contralateral dopamine-depleted groups. $A$, Dopamine tissue levels in caudate-putamen and nucleus accumbens (ng/mg). B, Net ipsilateral rotations in a 90 min test after $5 \mathrm{mg} / \mathrm{kg}$ amphetamine. $C$, Net ipsilateral rotations in a $30 \mathrm{~min}$ test after $0.1 \mathrm{mg} /$ $\mathrm{kg}$ apomorphine. All three assessment procedures indicate a greater that $98 \%$ depletion of striatal DA. ${ }^{* *}, p<0.01$.

consisted of $\mathrm{x}, \mathrm{y}$-coordinates, which were then used to draw reconstructions of movement trajectory and velocity profiles. The food tray, box, and food pellet were used as reference points (Whishaw et al., 1994).

\section{Procedure}

Pretraining. All rats were taught to reach for food from the food trays for $15 \mathrm{~d}$. They were free to use either limb. Their performance was scorcd in a 5 min test given at the end of this period. Over the next 30 $\mathrm{d}$, they were trained to reach for single food pellets in the filming box and again they were free to use either limb. If they showed a limb preference, the food pellet was placed in the receptacle contralateral to the used paw, as the pellet is easier to grasp from this location (Whishaw et al., 1986). They were then trained for a further $7 \mathrm{~d}$ with bracelets placed on their nonpreferred wrist. The bracelet prevents the paw from being advanced through the slot, but does not otherwise impede movements. After postsurgery, the rats attempted to use their good paw; the presurgical experience habituated them to this procedure (Whishaw et al., 1992a). When the rats were well trained, they were filmed from lateral, ventral, and frontal views.

Surgery. Once trained, the rats were divided into three groups: the control group $(n=4)$ received sham surgery, the contralateral group ( $n$ $=7$ ) rcccived a 6-OHDA lesion of the nigrostriatal bundle contralateral to the preferred limb, and the ipsilateral group $(n=7)$ received a 6-OHDA lesion of the nigrostriatal bundle ipsilateral to the preferred limb.

The rats were pretreated with pargyline $(50 \mathrm{mg} / \mathrm{kg}$, i.p.) and anesthetized with sodium pentobarbital $(50 \mathrm{mg} / \mathrm{kg}$, i.p.). Injections were $8 \mu \mathrm{g}$ of 6-OHDA in $4 \mu \mathrm{l}$ of vehicle $(0.9 \% \mathrm{NaCl}$ solution containing $0.1 \mathrm{mg} /$ $\mathrm{ml}$ ascorbate) infused over $5 \mathrm{~min}$ via a 30 gauge stainless steel cannula. Coordinates were $4.7 \mathrm{~mm}$ posterior to bregma, $1.8 \mathrm{~mm}$ lateral to the midline, and $7.4 \mathrm{~mm}$ ventral from the skull surface, with bregma and lambda horizontal.

Testing. The rats were given 5 min tests, from which hit percentage and number of reaches were scored, in the food tray task on days 1$15,30,60$, and 100 . They were videorecorded in the single pellet task and in the spontaneous grasping task on days $1,2,3,5,7,15,30$, and 60 . In addition, their performance in the single pellet task (hit percentage and number of reaches) was scored on day 60 , the day on which behavioral ratings were also done. Digit use was videorecorded and scored only on day 55 .

Neurochemical assay. The rats were killed by decapitation and their brains were rapidly removed and placed on an ice-cold surface. Immediately, a 6-8 mg punch of tissue was taken from the left and right dorsolateral striatum and nucleus accumbens in each brain. Tissue was placed into individual tubes containing $0.05 \mathrm{~N}$ perchloric acid and dihydroxybenzylamine (internal standard). The samples were homogenized and centrifuged at $1500 \times g$ for $4 \mathrm{~min}$. High-performance liquid chromatography (HPLC) with electrochemical detection was used to determine the concentration of DA in the samples (Castañeda et al., 1990). The supernatant was assayed for DA, DOPAC, HVA, and NE.

Statistical analysis. The results were subject to analysis of variance and follow-up $t$ tests (Winer, 1962). All of the behavioral ratings were performed prior to the neurochemical analysis, and the neurochemical analysis was performed by a neurochemist who was not aware of the animals' group assignment or behavioral performance.

\section{Results}

\section{Neurochemical and rotational analyses}

The 6-OHDA treatments produced an almost complete loss of DA from the dorsolateral striatum $[99.8 \%$ reduction, $F(2,33)=$ $81.8, p<0.001$ ] and nucleus accumbens [98.6\% reduction, $F(2,33)=111.2, p<0.001]$, relative to control rats (see Fig. 1). The concentrations DOPAC and HVA were reduced by over $95 \%$ in both structures as well $[F \mathrm{~s}(2,33)>20$, p's $<0.001]$. The concentration of NE was not statistically different in the dorsolateral striatum but was slightly reduced in the nucleus accumbens $[F(2,33)=9.1, p<0.01]$.

That the rats receiving 6-OHDA injections into the nigrostriatal bundle had DA depletions was confirmed by tests of rotation. The depleted rats had rotation scores toward the side ipsilateral to their lesions after amphetamine $[F(1,15)=6.8, p$ $<0.01]$ and toward the side contralateral to the lesion after apomorphine $[F(1,15)=6.75, p=0.019]$, indicative of extensive depletions (see Fig. 1) (Marshall and Ungerstedt, 1977; Hefti et al., 1980).

\section{Measures of reaches and successes}

Performance on the food tray task and single pellet task were similar and so quantification of performance was done each day for only the food tray task. Scoring on the single pellet task was done only for day 60 , the day on which behavioral ratings were done. This was a point well beyond whatever asymptotic recovery had occurred.

Food tray task. A summary of reaching success and number of attempts on daily tests in the food tray task is shown in Figure 2. Presurgery, there were no group differences in success. There were significant group differences postsurgery $[F(2,15)=115.84$, $p<0.001$ : control $>$ ipsilateral $>$ contralateral, $p$ 's $<0.05]$. The rats using their ipsilateral limb displayed improved success over the $100 \mathrm{~d}$ postsurgical period, but the other groups did not change [confirmed by a significant interaction between days and groups, $F(34,255)=2.4, p<0.05$, and follow-up tests on performance of the ipsilateral group across days, $F(17,255)-5.1$, $p<0.001]$. There were no group differences in the number of 
A. Success

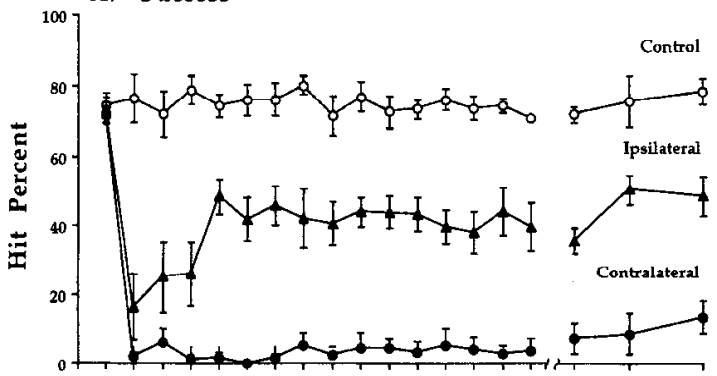

B. Attempts

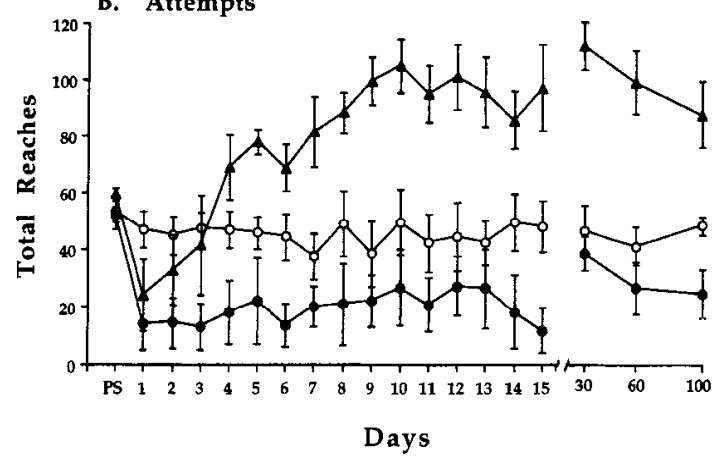

Figure 2. Reaching performance (mean and SE) by control and dopamine depleted rats using their ipsilateral-to-lesion limb (good limb) or contralateral-to-lesion limb (bad limb) presurgically (PS) and on tests given up to $100 \mathrm{~d}$ postsurgery in the food tray task. $A$, Percentage of successful reaches (hit Percent) on daily 5 min tests. $B$, Total number of reaches made on daily tests. Postoperatively, there were no significant changes in hit percentage and total reaches by the control and contralateral groups, although they differed from each other. The ipsilateral group showed significant postsurgical recovery in hit percentage and significant increase in the number of reaches, although they did not regain control levels of performance.

reaches made by the different groups presurgically. Postsurgically, there were differences $[F(2,15)=15.23, p=0.0002$ : ipsilateral $>$ control $>$ contralateral, $p<0.05$ ]. The rats using their ipsilateral limb showed increases in the number of reaches over the $100 \mathrm{~d}$ postsurgical period [confirmed by a significant group by days interaction, $F(34,255)=3.1, p<0.05$, and followup tests of the performance of the ipsilateral group across days, $F(17,255)=7.2, p<0.001]$. The other groups showed no change across days in either hit percent or number of reaches.

\section{Single pellet task}

Success. There was a significant group difference in reaching success $[F(2,15)=27.4, p<0.001$ : control $>$ ipsilateral $>$ contralateral; Fig. $3 A]$ and in numbers of reaches $[F(2,15)=$ 14.9, $p<0.001$ : ipsilateral $>$ control $>$ contralateral, $p<0.05$ ].

Limb component ratings. When control rats reach, the paw is lifted so that the palm is rotated inward, the digits are partially flexed, and digit tips are aligned along the midline of the body. The limb is then aimed by adduction of the elbow to align the forearm with the midline of the body. From this position the limb is advanced, and as it is advanced, the digits are opened. Once the paw reaches the food, it is pronated over the food by abduction of the elbow. Grasping is accomplished by closing the digits, and as the food is withdrawn, the paw is supinated by a movement about the wrist and by adduction of the elbow. The rat then sits back on its haunches to cat, and as it does so, the paw is further supinated to present the food to the mouth.
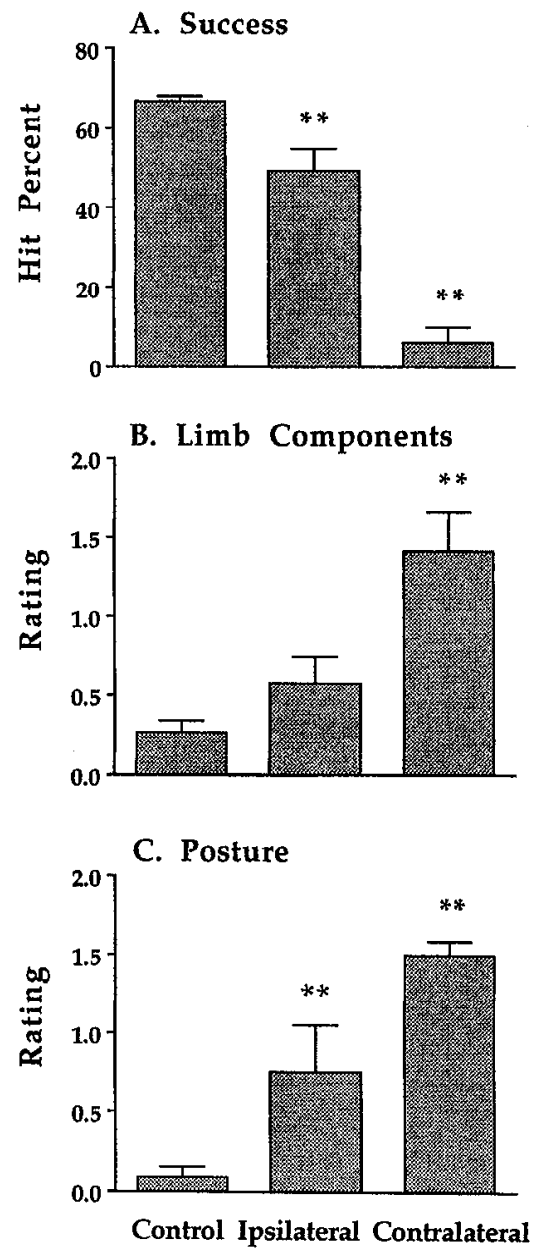

Figure 3. Reaching performance (mean and SE) by control and DAdepleted rats on tests given in the single pellet task at day 60 postsurgery. $A$, Percentage of successful reaches (hit Percent) on a 10 min test. $B$, Rating of 10 components movements of the limb. $C$, Rating of postural adjustments. ${ }^{* *}, p<0.01$; for the ratings: $0=$ normal, $1=$ mildly impaired, 2 = severely impaired.

The mean rating given to each of these 10 movements (day 60 postsurgery) is shown in Figure $3 B$. There were significant group differences in the rating scores of movement components comprising a reach $[F(2,15)=12.6, p<0.001$ : control $<$ ipsilateral $<$ contralateral, $p$ 's $<0.05$ ].

Follow-up analyses on the individual component movements showed that the ipsilateral group was significantly impaired relative to the control group in limb aiming, limb advancement, and food release $(p$ 's $<0.05)$. It was the raters' impression that these movements received high rating scores because the rats used peculiar postures (see below) rather than because of an inability to make the movements. The follow-up analyses indicated that the contralateral group was impaired, relative to the control group, on all component movements $(p$ 's $<0.05)$ except digit closure as the limb was lifted from the floor and digit opening as the limb was advanced. The rats advanced the limb diagonally toward the slot without aiming it and aligned the limb with the slot by ipsilateral-to-the reaching limb rotation of the body. The rats were impaired in pronation, and this combined with the diagonal trajectory of the paw invariably resulted in the food being missed. When food was grasped, the paw was not supinated, the food was poorly grasped, and it was 

A. Presurgery
B. Day 3
C. Day 60

Figure 4. Lateral view of paw and nose trajectories as the limb is advanced and withdrawn. $A$, Presurgery the advancing limb extends above and beyond the food pellet even though the point of initiation can be far from (top) or close to (bottom) the food shelf. After withdrawing the limb, food may be eaten close to the shelf (top) or farther away from it (bottom). $B$ and $C: T o p$, movements made when using the ipsilateral (good limb) are almost normal except that the rat usually eats the food while the paw is still on or close to the shelf. $B$ and $C$ : Bottom, The movement made by the contralateral (bad limb) is initially abbreviated $(B)$ and never regains the arching trajectory $(C)$. Food is nearly always ignored as the paw falls to the floor after being withdrawn from the shelf. Both preoperatively and postoperatively, the rats lift the nose so that the paw can advance and lower it when the paw is withdrawn. (The trajectories were digitized from video at 60 fields/ sec.)
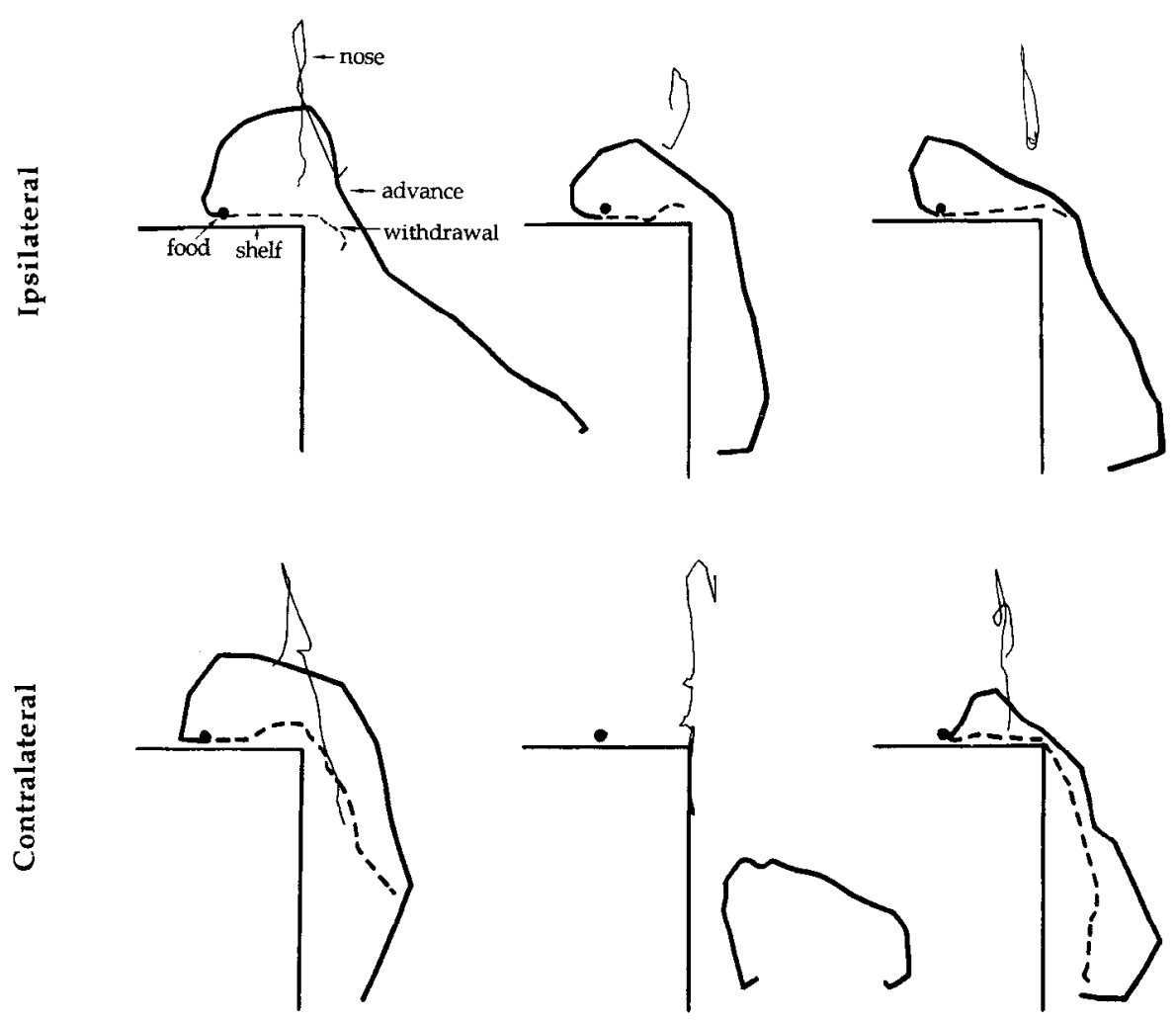

nearly always ignored as the paw was dropped to the floor after being withdrawn from the shelf.

Postural ratings. The control rats usually approached the slot directly or on only a very slight angle. They then sniffed the food pellet, lifted their head as they advanced the limb, and then advanced the limb, grasped, and withdrew the food with very little postural adjustment. If they missed the food on the initial advancement of the limb, they withdrew the limb and reached again without further postural adjustments. There were differences in the ratings received by the groups $[F(2,15)=21.4$, $p<0.001$ : control $<$ ipsilateral $<$ contralateral, $p<0.05 ;$ Fig. $3 C$. Follow-up tests indicated that the ipsilateral group had impaired rating scores for the angle of orientation to the food slot, for their posture when initiating a reach, and in their movements when withdrawing the food pellet $(p$ 's $<0.05)$. They frequently aligned themselves to the slot with a greater angle than did control rats, adjusted their posture before they reached, and they attempted to support themselves with the reaching paw after the food pellet was grasped. Rating scores for posture and movement during limb advancement were normal and they did not make more postural corrections during reaching than did control rats. The contralateral group had significantly impaired ratings on all five portions of reaching ( $p$ 's $<0.05)$. They aligned their body sideways to the slot, and made many postural adjustments during all phases of the reach. They attempted to use their good paw to aid reaching during all phases of the reach, and they frequently placed their good paw against the wall of the box or grasped the edge of the slot with it.

\section{Kinematic analysis of limb advancement}

Typical presurgical and postsurgical trajectories of advancement and withdrawal of the limb are shown in Figure 4. Prior to reach initiation, the nose is inserted into the slot to sniff the food and it is raised to allow the paw to advance to the food, and is lowered again as the paw is withdrawn. For control rats, the point of initiation can be far from (Fig. 4A, top) or close to (Fig. $4 A$, bottom) the food shelf and the advancing limb extends above and beyond the food pellet. The limb may be withdrawn just inside the shelf, where the food is passed to the mouth (Fig. $4 A$, top), or the rat might sit back on its haunches before it begins to eat (Fig. $4 A$, bottom). Postsurgically, a rat still sniffs the food and raises and lowers its nose to allow the limb to advance. The trajectory of limb advancement using the ipsilateral limb is almost normal (Fig. $4 B, C$, top), but upon grasping the food the rats begin to support themselves on the reaching paw. Consequently, in the early postsurgical period they often held their paw on the shelf as they grasped the food by mouth (day 3) but later in recovery they usually sat back on their haunches to eat (day 60). The trajectories made by the contralateral paw were consistently abnormal (Fig. 4B,C, bottom). Early after surgery the limb frequently did not reach the shelf (day 3), and thereafter even on successful reaches the arching trajectory was absent and the trajectory itself was irregular (day 60 ). When food was grasped, the paw was not supinated and fell to the floor after being withdrawn from the shelf, where the food was dropped (day 60 ).

To examine the aiming component of the reach (paw and elbow adduction), the digits and elbow were digitized in reference to the body outline. Control rats bring the digits to the midline of the body and then in an independent movement bring the elbow to the midline of the body. This movement "aims" the limb so that it can advance directly through the slot. As the limb advances, the elbow is abducted to pronate the paw over the food. This produces the typical digit and elbow trajec- 

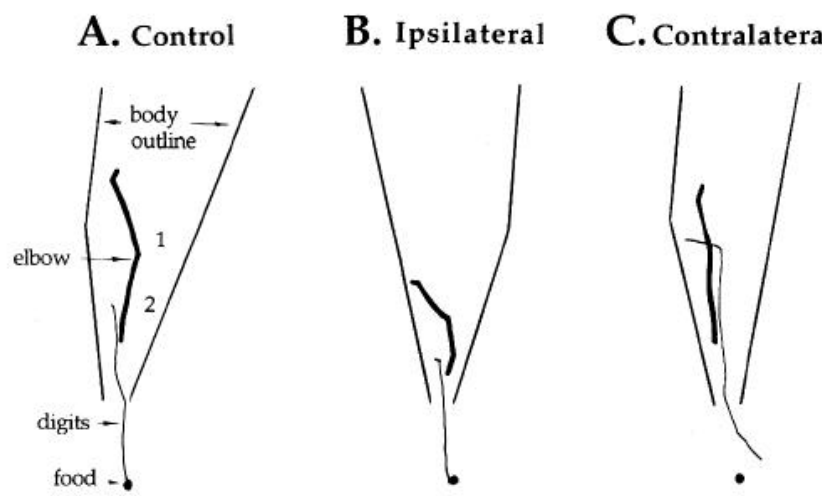

Figure 5. Ventral view of paw and elbow trajectories of the right limb during advancement to the food. $A$, Control rats first bring the digits to the midline of the body and then in an independent movement bring the elbow to the midline of the body (1). This movement "aims" the limb so that it can advance directly through the slot. As the limb advances, the elbow is abducted to pronate the paw over the food (2). $B$, The digit and elbow movements made by the ipsilateral-to-lesion limb of a DA-depleted rat are similar to those of a control rat. C, A DAdepleted rat reaching with the contralateral-to-lesion limb, advances the limb diagonally toward the slot and aligns the limb with the slot by ipsilateral-to-the-reaching-limb rotation of the body. Thus, they do not aim the limb by adducing the paw and elbow, and they do not pronate the paw by abducting the elbow. Note that the diagonal trajectory of the paw results in the food being missed. (The trajectories were digitized from video at 60 fields/sec.)

tory shown in Figure $5 \mathrm{~A}$. The digit and elbow movements made by the ipsilateral limb of a DA-depleted rat are similar to those of a control rat (Fig. $5 B$ ). The digit and elbow movements in rats using the contralateral limb are obviously abnormal (Fig. $5 C$ ) in that the elbow is not adducted to aim or abducted to pronate, and the limb takes a diagonal trajectory such that the paw bypasses the food (Fig. $4 C$ ).

\section{Digits movements}

When a control rat pronates its paw, the digits contact the shelf and food in an "arpeggio" pattern such that digit 5 (the outside digit) makes first contact followed successively by digits 4,3 , and 2. Digit 1 is rudimentary and is not used. The arpeggio pattern by which the digits are pronated over the food is produced by abduction of the elbow and movements about the wrist. The large food pellets are usually grasped with digits 5 , 4 , and 3 in what is best characterized as a whole paw grasp (Fig. $6 \mathrm{~A}$ ). The small pellets are grasped between digits 5 and 4 (Fig. $6 \mathrm{~B}$ ) and require more individual movement of the digits. As the digits grasp the food, the paw is quickly supinated as it is withdrawn from the shelf. The paw and digits movements made by the ipsilateral paw in a rat with a dopamine depletion are similar to those of control rats.

When DA-depleted rats use their contralateral limb, they usually drop the paw directly down onto the shelf. The absence of aiming and elbow abduction to pronate the paw appear to underlie the absence of the arpeggio digit pattern in contacting the shelf and food. If a large piece of food is contacted by the paw, the digits close in the whole-paw grasping pattern but then the paw is withdrawn without being supinated (Fig. $7 A$ ). If a small food pellet is contacted, grasping is much less effective, and although the pellet may be caught between the digits, it is raked off the shelf without digit closure or paw supination (Fig. 7B).
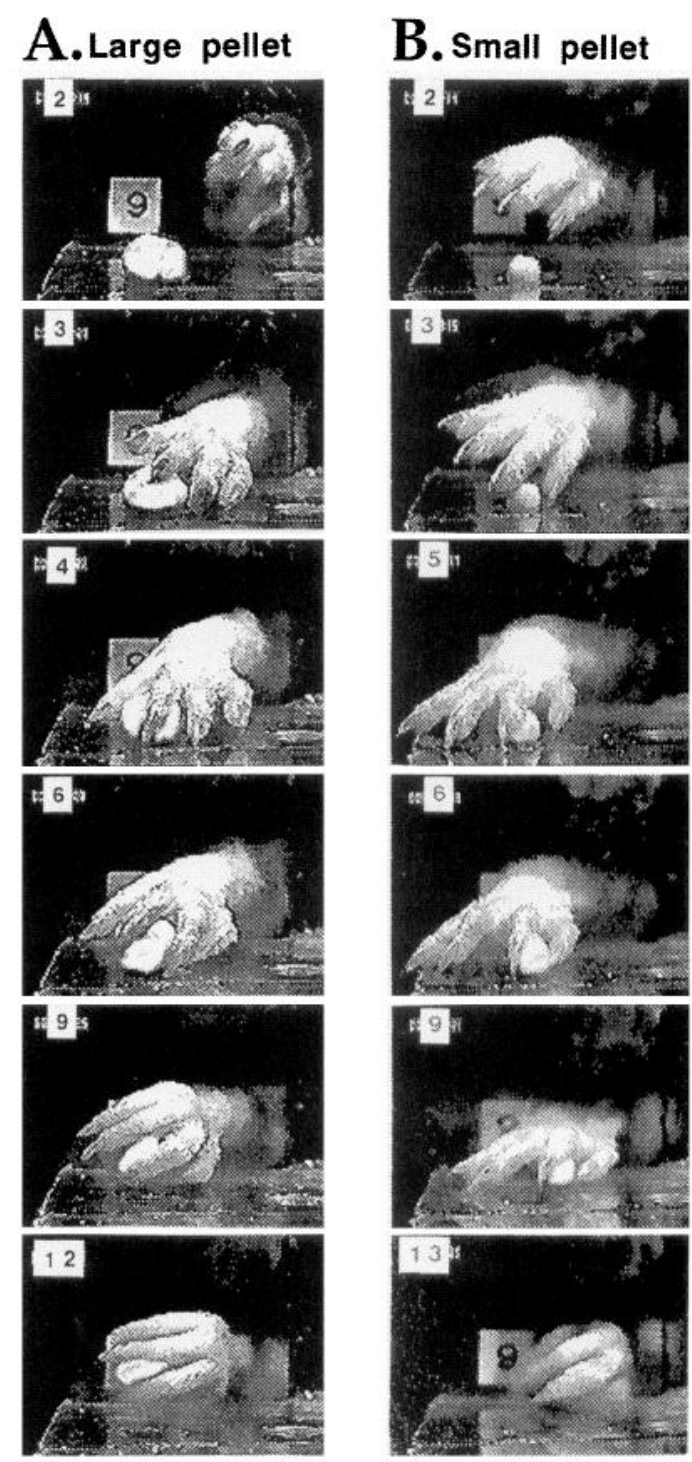

Figure 6. Paw and digit movements by a DA-depleted rat using its ipsilateral-to-lesion (good) limb to grasp a large food pellet $(A)$ or a small food pellet $(B)$. The movements are similar to those made by control rats. As the paw is pronated, the digits come into contact with the shelf and food in an "arpeggio" pattern such that digit 5 makes first contact followed successively by digits 4,3 , and 2 . The large food pellet is grasped with a whole paw grasp and the small pellet is grasped between digits 5 and 4 . Note that the paw is quickly supinated before being withdrawn from the shelf. Numbers in the left upper corner indicate video frames at 60 frames/sec.

\section{Compensatory adjustments and recovery}

When control rats initiate a reach, they use a diagonal supporting pattern, in which weight is supported on the nonreaching forelimb and the diagonal contralateral hind limb (Fig. 8A). Both supporting limbs actively assist weight shifts in that they aid movement of the body forward as the reaching limb advances and backward as it is withdrawn. Frequently, advancement of the reaching limb is accompanied by forward movement of the contralateral rear limb. This rear limb movement is made to anticipate the shift of weight to the hind limbs that occurs as the rat sits back to eat.

DA-depleted rats, reaching with their ipsilateral-to-lesion limb, support their weight and aid weight changes mainly with their 

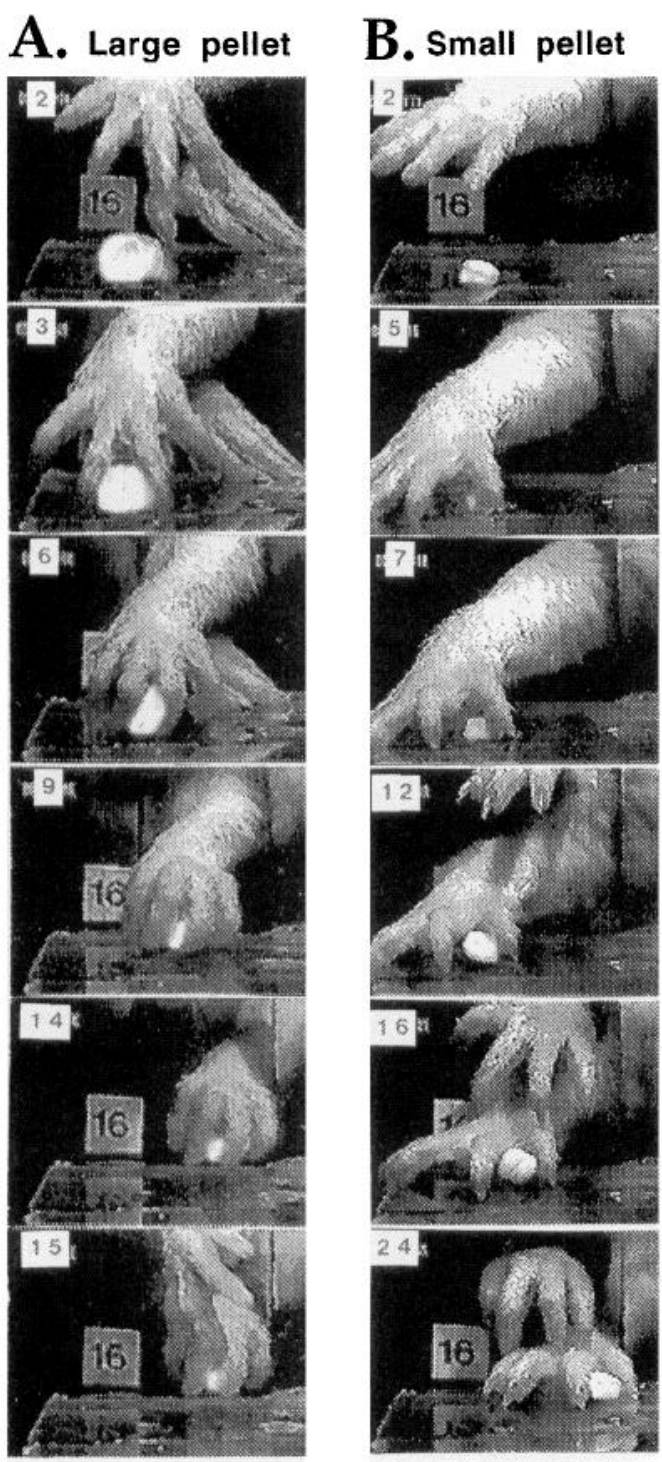

Figure 7. Paw and digit movements by DA-depleted rat using its contralateral-to-lesion (bad) limb to grasp a large food pellet $(A)$ or a small food pellet $(B)$. The rats do not aim the limb by adducting the paw and elbow (Fig. $4 C$ ). They do not pronate the paw over the pellet by abducting the elbow. The digits contact the shelf in a top-down movement without the "arpeggio" pattern. The large food pellet is grasped with a whole paw and the small pellet is raked back between digits 5 and 4 . Note that the paw is not supinated during withdrawal from the shelf. Numbers in the left upper corner indicate video frames at 60 frames/ sec.

ipsilateral rear limb. The contralateral rear limb plays a passive role in supporting weight, like a training wheel on a bicycle (Fig. $8 B$ ). Descriptively, the rat is standing and balancing itself on its ipsilateral limb. To aid balance, the tail is curved ipsilaterally and is pressed against the wall of the box. As the food pellet is grasped, the rat often transfers some support to the reaching limb and then pushes back with that limb in order to sit back with the food.

When rats use their contralateral limb to reach, weight is supported on both good limbs (Fig. $8 \mathrm{C}$ ). As the shelf is contacted with the advancing limb, weight is transferred onto this limb. This allows the rat to lift its good forelimb in an attempt to help grasp the food and/or retract the paw. When reaching with the contralateral limb, the tail is curved ipsilateral to the good side of the body, and pressed against the wall of the box for support and balance.

The patterns of postural compensatory adjustment, described above, take a number of days to develop postoperatively. Within the first few postsurgical days the rats attempt to use the normal diagonal supporting pattern. As the reach takes place, however, they appear to lose their balance and fall onto their contralateralto-lesion forelimb (Fig. 9A). After about 4-6 d postoperatively, however, the rats learn to balance on their ipsilateral-to-lesion limbs. Thereafter, they shift their weight throughout the course of the reach so that they maintain their balance on their good limbs (Fig. 9B).

\section{Spontaneous food eating}

When given a piece of food to eat, control rats pick up the food with the mouth and then sit back, transfer the food to the paws, and eat from the paws. They hold the food with the tips of the digits, the paws are cupped around the bottom half of the pellet, and the pellet is manipulated with both paws. Their sitting posture has their weight fairly equally supported on both hind limbs, with the limbs slightly asymmetrical in the anterior posterior direction (Fig. 10A). The DA-depleted rats supported their weight mainly on the good hind limb and tail. The contralateralto-lesion forepaw is used as a table to support the food pellet. During the first few postsurgical days the paw and forearm are supported on the floor and the head has a pronounced ipsiversive-to-the-bad-paw rotation, in part to balance weight and in part to gain access to the food pellet (Fig. 10B). After a number of postsurgical recovery days, the impaired paw, still serving as a table, is held above the floor and head and body rotation are much less pronounced. By this time, body weight is supported mainly on the good rear limb and balancing is aided by leaning against the wall of the cage (Fig. 10C). Postoperatively, the contralateral-to-lesion limb is seldom used to manipulate the food, and the digits are almost never observed to be involved in manipulating the food.

\section{Discussion}

The results of this study demonstrate that rats with unilateral DA depletions have severe impairments in making voluntary movements with the limbs contralateral to the lesion, both for reaching and for actively adjusting posture when reaching. These impairments account for their inability to make successful reaches with the forelimb contralateral to the lesion and they contribute to the impaired performance observed with the limb ipsilateral to the lesion. To compensate for the impairments in their contralateral limbs, they rely on the ipsilateral limbs to actively adjust support.

Rats using their contralateral-to-lesion limb made few successful reaches in either an easy or difficult reaching task, confirming other work (Whishaw et al., 1986). A video analysis of how the limb was used found that component movements of the reach, including aiming, pronating, and supinating the paw, were largely absent. Rather, the limb was advanced on a diagonal trajectory and a variety of postural and whole body movements assisted moving the limb toward the food. These results suggest that DA depletion results in the loss of certain "fractioned" components of the reaching movements while sparing the ability to advance and withdraw the limb. In addition, high-speed video recording showed that the pattern of digit use for food grasping was abnormal. The impairments in limb and digit use are very 

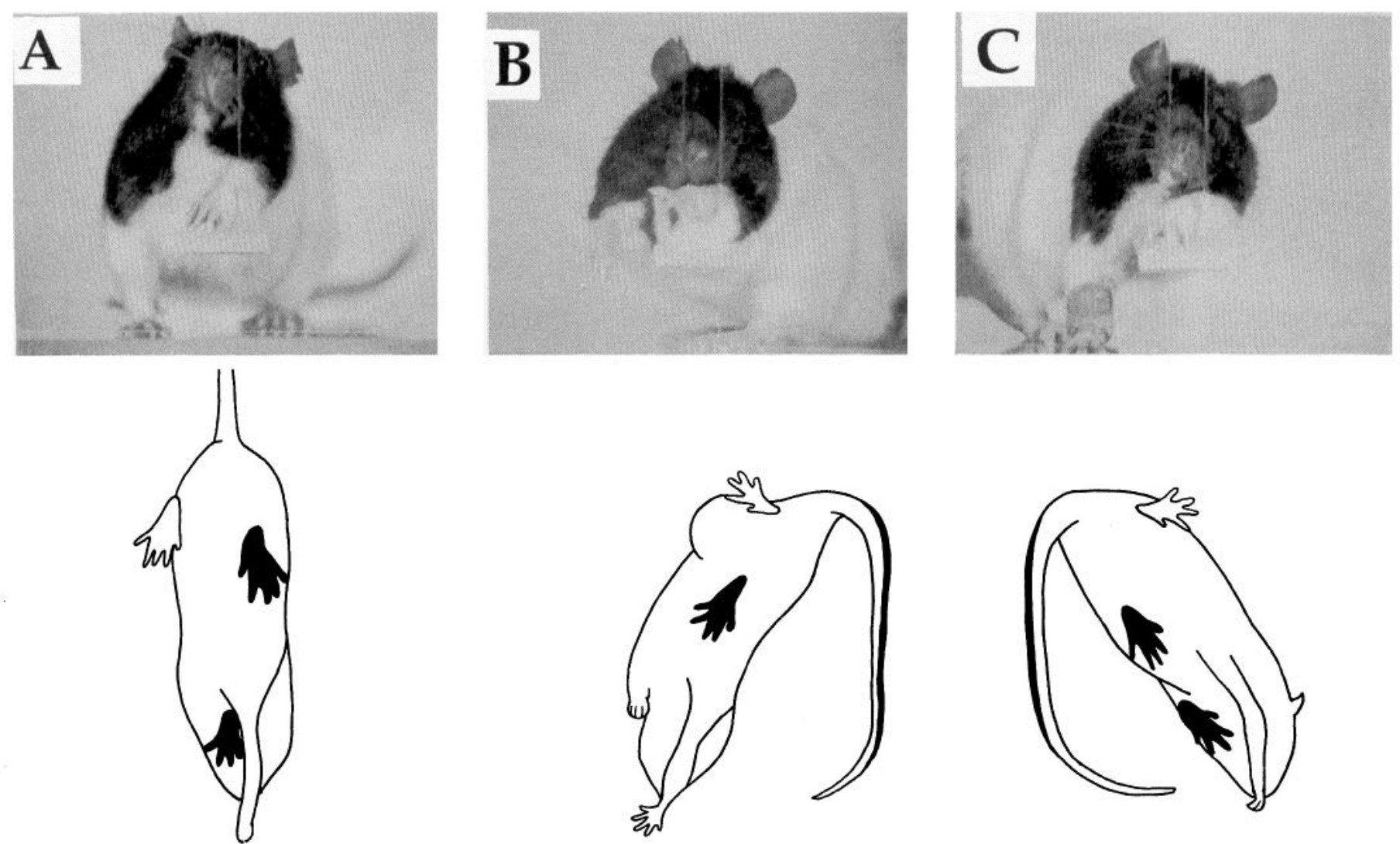

Figure 8. Body weight distribution during reaching. Top, Posture as a food pellet is grasped. Bottom, Shaded paws indicate main weight-bearing limbs. $A$, A control rat uses a diagonal supporting pattern in which weight is supported on the nonreaching forelimb and the diagonal contralateral hindlimb. As the rat reaches the nonweighted hind limb is moving forward (top). During withdrawal, weight is shifted to the hind limbs, and the nonreaching forelimb plays an active role in shifting weight to the ipsilateral hind limb. $B$, When a DA-depleted rat uses its ipsilateral-to-lesion (good) limb to reach, weight is supported mainly on the ipsilateral hind paw. As the food pellet is grasped, weight is transferred onto the reaching forelimb. The food is often eaten as the paw remains on the shelf or the rat shifts the weight of the forequarters onto the ipsilateral hind limb to adopt sitting position. Note that the tail is curved ipsilateral to the good side of the body, and is pressed against the wall of the box (shading) to support and balance the body. $C$, When a DA-depleted rat reaches with its contralateral-to-lesion (bad) paw, weight is supported on both good limbs. As the reach takes place and the shelf is contacted, weight is transferred to the reaching limb. Note that the tail is curved ipsilateral to the good side of the body, and is pressed against the wall of the box (shading) to support and balance the body.

similar to that following damage to cortical systems (Whishaw et al., 1991, 1993), suggesting that the neocortex and striatum have a cooperative role in producing certain aspects of limb movements. Additionally, however, the impairment following DA depletion, seems much more severe than that following damage to cortical systems, suggesting that there are at least some separate contributions made by cortical and striatal structures (DeLong et al., 1984).

It is known that there are DA projections to the neocortex (Herrera-Marschitz et al., 1989), and it is possible that damage to this projection could contribute to the deficits described here. It is difficult to assess this idea, however, because attempts to selectively deplete DA in rat motor cortex have not been successful (Whishaw et al., 1992a). Nevertheless, available evidence suggests that the severe impairments in reaching are attributable to the depletion of dopamine in the striatum (Siegfried and Bures, 1980; Evenden and Robbins, 1984; Sabol et al., 1985; Whishaw et al., 1986, 1992a). Until such time that DA can be selectively depleted in motor cortex, the contribution of the cortical projection to reaching cannot be unambiguously determined.

The results also confirmed that the rats were impaired in use of their ipsilateral-to-lesion limb in reaching for food but showed substantial recovery over the postoperative test period (Whishaw et al., 1986, 1992a). Analysis of limb and digit movements suggested that this impairment did not stem from an inability to make the normal components of the movements or to use normal paw and digit movements in grasping. It was found that the rats adopted peculiar postures during some phases of the reach, and it seems likely that the postural abnormalities contributed to the reaching impairment (see below). The postural abnormalities likely also contributed to the increased number of reaches that they displayed, because attempting to balance on the ipsilateral limb (see below), they would make more misses, for which they compensated by making additional reaches. It is interesting in this respect that rats reaching with their ipsilateral limb in the "staircase" task, in which rats lie on a platform and reach down onto a shelf, have normal reaching success scores (Abrous et al., 1992). Here, only the trunk of the rat is supported on the platform, and this artificial support could scrve as a crutch to reaching.

Most of the voluntary movements performed by a standing animal or human are accompanied by postural adjustments that ensure that the center of gravity and equilibrium are maintained 
A. Day 3


bad side

reaching paw

\section{B. Day 60}
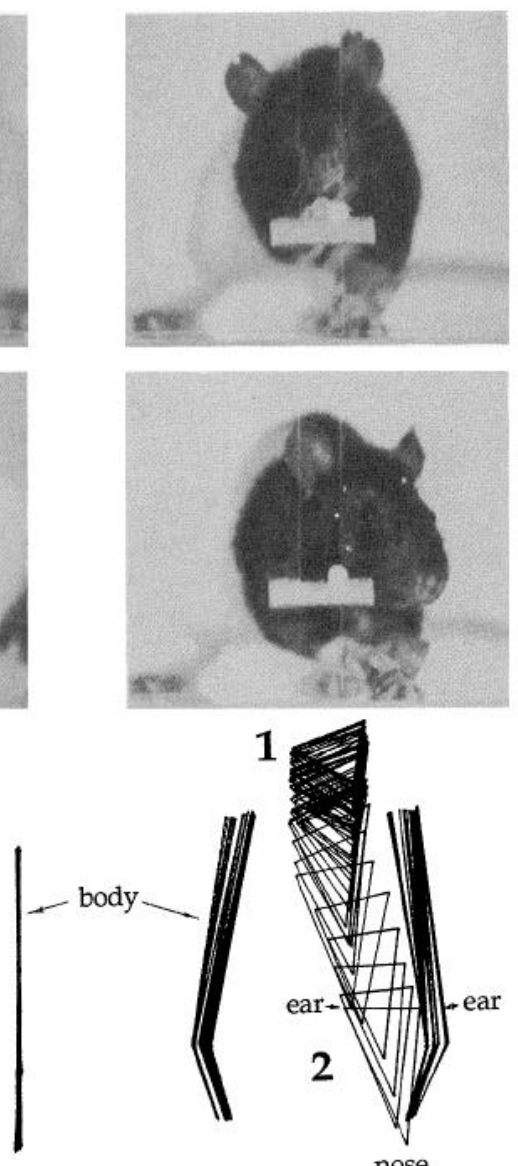

nose
Figure 9. Body weight distribution changes on day $3(A)$ and day 60 $(B)$ of rats reaching with contralateral-to-lesion (bad) limbs. $A$, On day 3 , as illustrated by head position relative to the body, the rats shift its weight to the bad side from the beginning (1) to the end (2) of the reach. $B$, By day 60 , the rat shifts its weight to its good side to maintain balance. The triangles represent the head (two ears and nose) and they show the successive displacement of the head from its initial ( 1 and top photo) to terminal ( 2 and bottom photo) position. The vertical lines represent the body outline and show the shift in body position through the course of the reach.

during movement. Usually, when an animal is standing, its center of gravity is shifted slightly forward (Frolov et al., 1988; Ioffe et al., 1988). If a forelimb is lifted without any postural adjustment, the animal will fall. Therefore, an anticipatory postural adjustment shifts the animal's center of gravity within the area defined by the remaining supporting limbs. In making this shift in the center of gravity, the animals use a "diagonal" postural supporting pattern, unloading the limb diagonally opposite to the lifted one and loading the other pair of limbs (Gahery et al., 1980; Ioffe et al., 1988). The postural adjustment that shifts the center of gravity is not simply passive but is active and anticipatory.

In this study it was found that normal rats adopt the diagonal postural pattern in anticipation of moving the reaching limb. Thus, they support their weight on the contralateral forelimb and the ipsilateral hind limb. Very often, as the reaching limb was advanced, the contralateral hind limb was lifted and ad- vanced at the same time, in anticipation of the postural adjustment that the rat would make when it sat back to eat the food. Consequently, during the course of limb advancement, the rat may have only its two diagonally supporting limbs on the floor. The two supporting limbs bear the animal's body weight and they actively shift the rat's body forward as it reaches and backward again as it retrieves the food. Thus, the weight bearing limbs not only support weight but actively participate by moving the body during the reach.

The rats that received unilateral DA depletions did not use the diagonal supporting pattern, either when reaching with their contralateral-to-lesion forelimb or their ipsilateral forelimb. Rather, they attempted to use their good limbs in a supporting function. Thus, when using their contralateral-to-lesion forelimb, weight was supported mainly on the ipsilateral forelimb and hindlimb in a "unilateral" pattern. When reaching with the ipsilateral-to-lesion forelimb, body weight was supported mainly on the ipsilateral rear limb, a "monolimb" pattern. In both cases, the remaining rear bad limb was used mainly as a "training wheel" for balance. It is unlikely that these supporting postures are adopted because the contralateral-to-lesion limbs are unable to support weight, since rats with bilateral DA depletions use their limbs to support weight and defend equilibrium (Schallert et al., 1978, 1979). It is more likely that the rats need to adjust their posture during the reach and can do so only with the ipsilateral-to-lesion limbs. Thus, they modify their posture from the diagonal pattern to a pattern that allows them to move from the good limbs. It is important to note that adoption of the unilateral and monolimb supporting strategies is facilitated by the tail, which contributes to support and balance.

In the early postsurgical period the rats with DA depletions did not use the alternative supporting strategies especially well. By day 4 postoperatively, the alternative supporting postures began to appear and thereafter they became increasingly used. Paralleling the emergence of the new supporting patterns, the rats began to show improved reaching and spontaneous food handling ability. For the rats using the contralateral limb this improvement was largely qualitative, but for the rats using the ipsilateral limb there were significant improvements in reaching success. This relationship suggests that the novel supporting patterns serve an effective compensatory function.

The results of the present study provide some novel insights in the way in which loss of dopamine impairs movement in that they suggest that the ability to make voluntary movements both in skilled reaching and for adjusting support is seriously compromised (see also Schallert et al., 1979). They also indicate that increasing reliance is placed on the unimpaired limbs, allowing them to contribute in a compensatory role. We suggest, and preliminary investigations confirm, that similar compensatory adjustments are made during other behaviors including spontaneous walking, turning, and conspecific interactions. It is also interesting that Herrera-Marschitz et al. (1990) have reported scoliosis in unilateral DA-depleted rats and suggest that it is a consequence of increased muscle tone on the contralateralto-lesion side of the body. Here we suggest a contributory influence may be the asymmetric posture and movement strategy that was found to characterize the behavior of the rats. Finally, although it is known that a variety of neural changes can take place to compensate for DA loss (Robinson et al., 1990; Zigmond et al., 1990), the present study clearly shows that compensatory behavioral changes can contribute to recovery processes in an important way. 


\section{A. Presurgery}
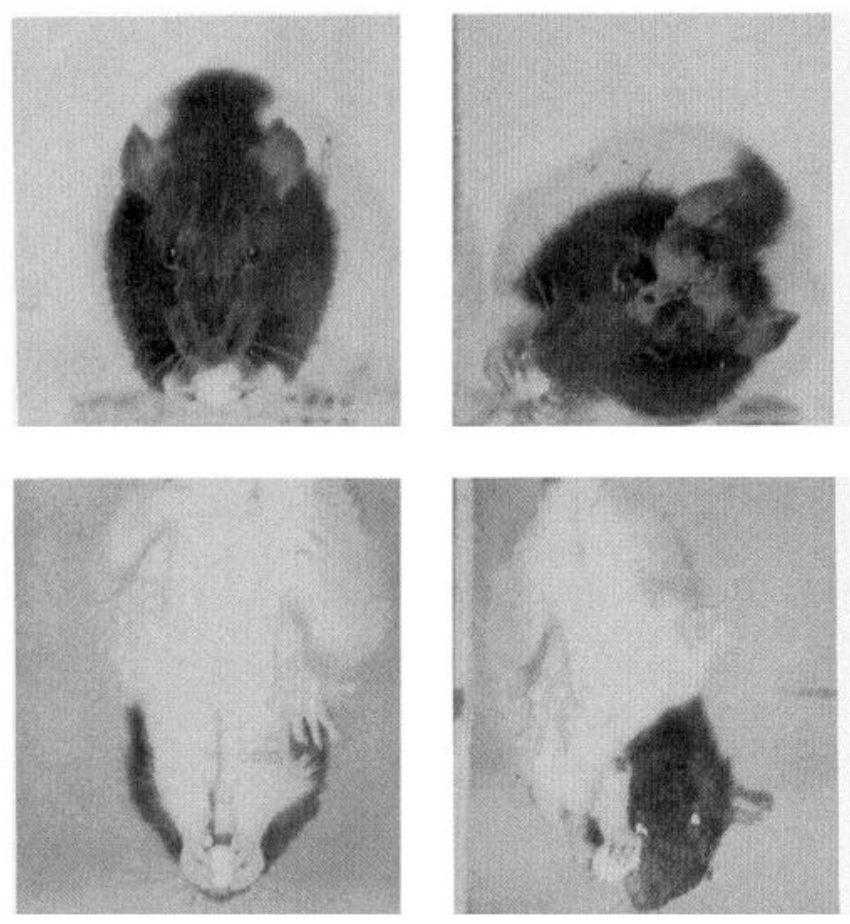

\section{Day 60}
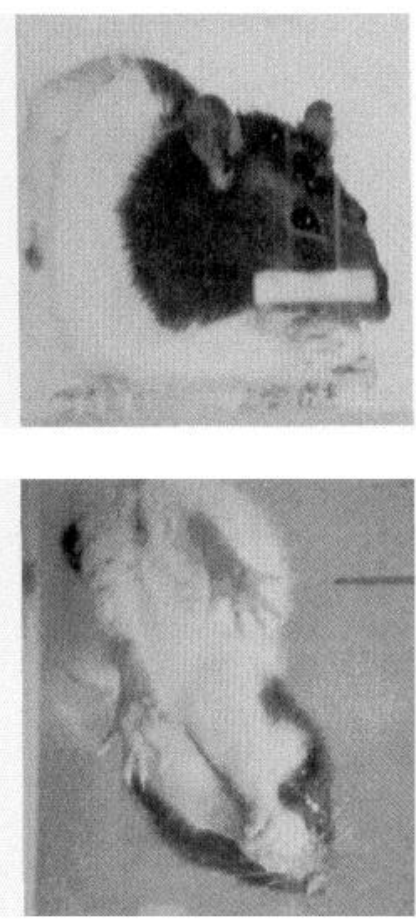

Figure 10. A unilateral DA-depleted rat (right side depletion) eating a $1 \mathrm{gm}$ food pellet. $A$, The control posture has the limbs slightly asymmetrical in the anterior-posterior direction with weight fairly equally supported on both hind limbs. The paws are cupped around the bottom half of the pellet and the pellet is manipulated with both paws. $B$, Three days postsurgery the ipsilateral-to-lesion "bad" forepaw is used as a table to support the food pellet, and it in turn is supported on the floor. The head has a pronounced ipsiversive rotation, in part to balance weight and in part to gain access to the food pellet. $C$, Day 60 postsurgery, weight is supported mainly on the ipsilateral-to-lesion "good" hindlimb and balancing is assisted by leaning against the wall of the box. The contralateral-to-lesion "bad" forelimb is still used as a table, but is now no longer supporting body weight by contacting the floor.

\section{References}

Abrous DN, Wareham AT, Torres EM, Dunnett SB (1992) Unilateral dopamine lesions in neonatal, weanling and adult rats: comparison of rotation and reaching deficits. Behav Brain Res 51:67-75.

Bennett KMB, Adler CH, Stelmach GE, Castiello U (1993) A kinematic study of the reach to grasp movement in a subject with hemiparkinson's disease. Neuropsychology 31:709-716.

Carli M, Evenden JL, Robbins TW (1985) Depletion of unilateral striatal dopamine impairs initiation of contralateral actions and not sensory attention. Nature 313:679-682.

Castañeda E, Whishaw IQ, Lermer L, Robinson TE (1990) Dopamine depletion in neonatal rats: effects on behavior and striatal dopamine release assessed by intracerebral microdialysis during adulthood. Brain Res 508:30-39.

DeLong MR, Alexander GE, Georgopoulos AP, Crutcher MD, Mitchell SJ, Richardson RT (1984) Role of basal ganglia in limb movements. Hum Neurobiol 2:235-244.

Eshkol N, Wachmann A (1958) Movement notation. London: Weidenfeld and Nicholson.

Evenden JL, Robbins TW (1984) Effects of unilateral 6-hydroxydopamine lesions of the caudate-putamen on skilled forepaw use in the rat. Behav Brain Res 14:61-68.

Frolov AA, Birjukova EV, Ioffe ME (1988) On the influence of movement kinematics on the support pressure pattern during postural adjustment of quadrupeds. In: Stance and motion: facts and concepts (Gurfinkel VS, Ioffe ME, Masson J, Roll JP, eds), pp 227-238. New York: Plenum.

Gahery Y, Ioffe ME, Masson J, Polit A (1980) The postural support of movement in cat and dog. Acta Neurobiol Exp (Warsz) 40:741756.

Hamilton MH, Garcia-Munoz M, Arbuthnott GW (1985) Separation of the motor consequences from other actions of unilateral 6-hydroxydopamine lesions in the nigrostriatal neurones of rat brain. Brain Res 348:220-228.

Hefti F, Melamed E, Sahakian BJ, Wurtman RJ (1980) Circling behavior in rats with partial unilateral nigrostriatal lesions: affect of amphetamine, apomorphine and dopa. Pharmacol Biochem Behav 12:185-188.
Herrera-Marschitz M, Goiny M, Utsumi H, Ungerstedt U (1989) Mesencephalic dopamine innervation of the frontoparietal (sensorimotor) cortex of the rat: a microdialysis study. Neuroscience 97:266-270.

Herrera-Marschitz M, Utsumi H, Ungerstedt U (1990) Scoliosis in rats with experimentally-induced hemiparkinsonism: dependence upon striatal dopamine denervation. J Neurol Neurosurg Psychiatry 53: 39-43.

Ioffe ME, Ivanova NG, Frolov AA, Birjukova EV, Kiseljeva NV (1988) On the role of motor cortex in the learned rearrangement of postural coordinations. In: Stance and motion: facts and concepts (Gurfinkel VS, Ioffe ME, Masson J, Roll JP, eds), pp 213-226. New York: Plenum.

Marshall JF, Ungerstedt U (1977) Supersensitivity to apomorphine following destruction of the ascending dopamine neurons: quantification using the rotational model. Eur J Pharmacol 41:361-367.

Marshall JF, Richardson JS, Teitelbaum P (1974) Nigrostriatal bundle damage and the lateral hypothalamic syndrome. J Comp Physiol Psychol 87:808-830.

Martin JP (1967) The basal ganglia and posture. London: Pittman.

Ranje C, Ungerstedt U (1977) Lack of acquisition in dopamine denervated animals tested in an underwater maze. Brain Res 134:95111.

Robinson TE, Castañeda E, Whishaw IQ (1990) Compensatory changes in striatal dopamine neurons following recovery from injury induced by 6-OHDA or methamphetamine: a review of evidence from microdialysis studies. Can J Psychol 44:253-275.

Sabol KE, Neill DB, Wages SA, Church WH, Justice JB (1985) Dopamine depletion in a striatal subregion disrupts performance of a skilled motor task in the rat. Brain Res 335:108-120.

Sacks OW (1973) Awakenings. London: Duckworth.

Schallert T, Hall S (1988) 'Disengage' sensorimotor deficit following apparent recovery from unilateral dopamine depletion. Behav Brain Res 30:15-24.

Schallert T, Whishaw IQ, DeRyck M, Teitelbaum P (1978a) The postures of catecholamine depletion catalepsy: their possible adaptive value in thermoregulation. Physiol Behav 21:817-820.

Schallert T, Whishaw IQ, Ramirez VD, Teitelbaum P (1978b) Compulsive, abnormal walking caused by anticholinergics in akinetic, 6-hydroxydopamine-treated rats. Science 199:1461-1463. 
Schallert T, DeRyck M, Whishaw IQ, Ramirez VD, Teitelbaum P (1979) Excessive bracing reactions and their control by atropine and $l$-dopa in an animal analog of Parkinsonism. Exp Neurol 54:33-43.

Schallert T, Upchurch M, Lobaugh N, Farrar SB, Spirduso WW, Wilcox RE (1982) Tactile extinction: distinguishing between sensorimotor and motor asymmetries in rats with unilateral nigrostriatal damage. Pharmacol Biochem Behav 16:455-462.

Schultz Q (1982) Depletion of dopamine in the striatum as an experimental model of Parkinsonism: direct effects and adaptive mechanisms. Prog Neurobiol 8:121-166.

Siegfried B, Bures J (1980) Handedness in rats: blockade of reaching behavior by unilateral 6-OHDA injections into substantia nigra and caudate nucleus. Physiol Psychol 8:360-368.

Uguru-Okorie DC, Arbuthnott GW (1981) Altered paw preference after unilateral 6-hydroxydopamine injections into lateral hypothalamus. Neuropsychology 19:463-467.

Ungerstedt U (1971) Adipsia and aphagia after 6-hydroxydopamine induced degeneration of the nigro-striatal dopamine system. Acta Physiol Scand [Suppl] 367 82:96-122.

Ungerstedt U, Arbuthnott GW (1970) Quantitative recording of rotational behavior in rats after 6-hydroxy-dopamine lesions of the nigrostriatal dopamine system. Brain Res 24:485-493.

Whishaw IQ, Dunnett SB (1985) Dopamine depletion, stimulation or blockade in the rat disrupts spatial navigation and locomotion dependent upon beacon or distal cues. Behav Brain Res 18:11-29.

Whishaw IQ, Pellis SM (1990) The structure of skilled forelimb reaching in the rat: a proximally driven movement with a single distal rotatory component. Behav Brain Res 41:49-59.

Whishaw IQ, Tomie J (1988) Food wrenching and dodging: a neuroethological test of cortical and dopaminergic contributions to sensorimotor behavior in the rat. Behav Neurosci 102:110-123.
Whishaw IQ, O'Connor WT, Dunnett SB (1986) The contributions of motor cortex, nigrostriatal dopamine and caudate-putamen to skilled forelimb use in the rat. Brain 109:805-843.

Whishaw IQ, Pellis SM, Gorny BP, Pellis VC (1991) The impairments in reaching and the movements of compensation in rats with motor cortex lesions: an endpoint, videorecording, and movement notation analysis. Behav Brain Res 42:77-91.

Whishaw IQ, Castañeda E, Gorny BP (1992a) Dopamine and skilled limb use in the rat: more severe bilateral impairments follow substantia nigra than sensorimotor cortex 6-hydroxydopamine injection. Behav Brain Res 47:89-92.

Whishaw IQ, Dringenberg HC, Pellis SM (1992b) Spontaneous forelimb in free feeding by rats: motor cortex aids limb and digit positioning. Behav Brain Res 48:113-125.

Whishaw IQ, Pellis SM, Gorny BP, Kolb B, Tetzlaff W (1993) Proximal and distal impairments in rat forelimb use in reaching follow unilateral pyramidal tract lesions. Behav Brain Res 56:59-76.

Whishaw IQ, Gorny BP, Tran-Nguyen LTH, Castañeda E, Miklyaeva EI, Pellis SM (1994) Making two movements at once: impairments of movement, posture, and their integration underlie the adult skilled reaching deficit of neonatally dopamine-depleted rats. Behav Brain Res, in press.

Winer BJ (1962) Statistical principles in experimental design. New York: McGraw-Hill.

Zigmond MJ, Stricker EM (1973) Recovery of feeding and drinking by rats after intraventricular 6-hydroxydopamine or lateral hypothalamic lesions. Science 182:717-720.

Zigmond MJ, Abercrombie ED, Berger TW, Grace AA, Striker EM (1990) Compensations after lesions of central dopaminergic neurons: some clinical and basic implications. Trends Neurosci 13:290-296. 